\title{
Cascading blackout overall structure and some implications for sampling and mitigation
}

\author{
Ian Dobson ${ }^{\mathrm{a}, *}$, David E. Newman ${ }^{\mathrm{b}}$ \\ ${ }^{a}$ ECpE department, Iowa State University, Ames IA 50011 USA \\ ${ }^{b}$ Physics department, University of Alaska, Fairbanks AK 99775 USA
}

\begin{abstract}
Cascading blackouts can be thought of as initiating events followed by propagating events that progressively weaken the power system. We briefly discuss the implications for assessing cascading risk by proper sampling from the various sources of uncertainty and for mitigating cascading risk by reducing both the initiating events and their propagation.
\end{abstract}

Keywords: Power transmission reliability, risk analysis, simulation

\section{Introduction}

Cascading failure can be defined as a sequence of dependent events that successively weakens or degrades the power system [1]. The events are often individual power system components being outaged or damaged or misoperating, but can also include a device functioning as designed but nevertheless contributing to the cascade, or adverse actions by software, automatic controls, or operators $[2,3,4,5,6]$. This short paper describes the overall structure of cascading and the various sources of uncertainty in order to foster more comprehensive modeling and mitigation of cascading blackouts.

As shown in Fig. 1, cascading failure starts with a primary or "trigger" event and proceeds with further secondary events. All the events interact with the

\footnotetext{
*Corresponding author is Ian Dobson; telephone 15152940922

Email addresses: dobson@iastate.edu (Ian Dobson), denewman@alaska.edu (David E. Newman)
}

Preprint submitted to Elsevier

July 10, 2016

(C) 2016. This manuscript version is made available under the Elsevier user license http://www.elsevier.com/open-access/userlicense/1.0/ 


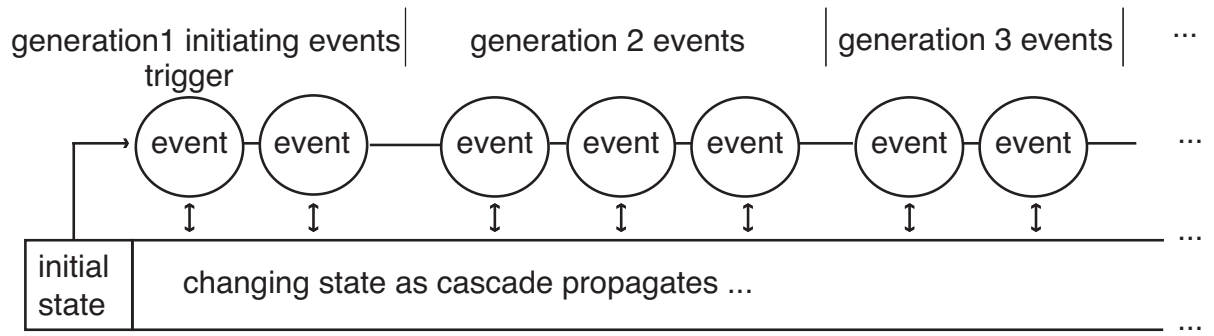

Figure 1: Overall structure of cascading failure in which events are grouped into successive generations. A trigger event followed by immediate protection actions forms an initiating generation of events. Subsequent generations of cascading events can follow, and the system state changes and weakens as the cascading events propagate.

system state as the cascade proceeds. The occurrence of each event depends on the system state, the system state is affected by every event that has already occurred, and the system state degrades throughout the cascade. The progressive weakening or degradation of the system as the cascade propagates is characteristic of cascading failure $[2,3,6,7]$. The system state includes such factors as which components are in service, component loadings, which control modes and operational schemes are active, generation margin, hidden failures, and situational awareness.

Substantial cascading events are rare because the initial system state is usually robust enough that it withstands the first few events and the cascade stops. But in an unfavorable initial system state, a trigger event can lead to many further events that become a substantial cascade and blackout. The progressive degradation of the system as the cascading events progress make it much more likely in each stage of the cascade that there are further cascading events than if the events were independent $[8,9,10] .{ }^{1}$ There is a small but significant probability of a long series of cascading events, and the probability distribution of observed cascading size has a "heavy tail" or "power law region" that implies a substantial risk of occasional large blackouts $[11,12,13,14]$.

\footnotetext{
${ }^{1}$ Not all events during cascades are dependent on the previous events; unrelated outages can occur and can have either substantial or minimal effects on the subsequent cascading. Data analysis in [10] estimates that about $6 \%$ of propagating outages in cascades are unrelated.
} 


\section{Overall cascade structure}

It is useful to divide the cascading events into initiating and propagating events:

$$
\text { cascading }=\text { initiating events then propagation }
$$

The trigger event may immediately cause further events, which, together with the trigger event itself, form the initiating events (for example, see the protection control groups in [15]). The propagating events are any events following the initiating events. It is convenient to think of any series of events as a cascade $[16] .^{2}$ Many cascades stop quickly so that there are no propagating events [10]. Examples of trigger events include short circuits due to lightning or tree contacts or animals, severe weather, earthquakes, operational or planning errors, equipment failure, or vandalism.

Making the distinction between triggering events and propagating events is useful because they have different mechanisms and analyses. The triggers are random failures often occurring at random times with no preceding cause within the power system, whereas the propagating events arise jointly from the preceding events and the changing power system state. The statistics of the trigger events ${ }^{3}$ follow from standard risk analysis [17], whereas the propagating events are much more complicated and probability models for their analysis are only starting to emerge $[7,16,9]$.

It is sometimes useful to group the events into generations. For example, if the timing of events is available, then events following each other within the fast timescale of automatic protection actions or that cannot be distinguished from simultaneous events due to the time discretization can be grouped into the same generation [16]. Another example is that simulations often produce

\footnotetext{
${ }^{2}$ For assessing cascading probability, it is just as important to consider the events that do not cascade further as the events that do cascade further. Focusing only on the multiple cascading events would strongly skew any statistics towards unreliability.

${ }^{3}$ The initiating events that are not trigger events arise in various ways with amenability to known risk analysis as described in [18].
} 
multiple events in each "pass" of the simulation that can be grouped together [19]. The first generation of the cascade is the initiating events, and the subsequent generations are groupings of the propagating events. In many observed blackouts the events happen more quickly later in the cascade [20] and some researchers suggest dividing the cascading into slow cascading followed by fast cascading [21].

Some evidence for the overall cascading structure asserted in this section is provided by the validation with real blackout data of models that include this structure $[16,22]$.

\section{Implications of Cascade Structure}

This section shows how the cascade structure determines how cascading simulations should sample from uncertainty and how cascades can be mitigated. Sampling from the uncertainties in each of the initial state, the trigger event, and the progress of the cascade is indicated. Mitigation of cascading should not only address the initiating events and the small blackouts, but also the cascade propagation and large blackouts. In the longer term, complex system considerations shape the effects of mitigation as the power system evolves.

\subsection{Sampling in cascading simulations}

The structure of cascading and the various sources of uncertainty affect how simulations should sample or select the cases to be run in order to assess cascading risk. Each cascade is strongly and jointly influenced by the initial system state and the trigger event. For example, a given trigger event may lead to further cascading events in only a few of the plausible initial system states. And different system states are either invulnerable to cascading or vulnerable to cascading with different triggers. Another example is that a given cascade might stop at the fourth event when the cascade starts from some initial system state and continues past the fourth event when the cascade starts from another initial system state because of differences that affect the threshold condition for 
the fifth event. It follows that trigger events and initial system states must be jointly sampled for each simulated cascade.

The threshold conditions for further outages are typically complicated functions of the previous events and the state, and it is often useful to model probabilistically the condition for a further outage of a given component and the progress of the cascade. Indeed, similar initiating events under similar conditions can propagate differently on different occasions in the real power system. The simulation should sample from the uncertainties in the system state, the trigger events, and the progress of the cascade. These comments also apply to selecting the initial system states and trigger events of simulations that model the cascade evolution deterministically. It is unrealistic to simulate the same cascade very many times, and the sampling from the uncertainties provides a realistic variety of cascades. Moreover, the uncertainties should be sampled in an unbiased way across the full ranges of uncertainties in order to properly estimate the probabilities and risks of cascading.

A significant exception to the sampling requirements is using simulation to reproduce a particular blackout that has already occurred. In this case, the initial state and trigger events are known, and the simulation thresholds and models can be skillfully tuned to reproduce the observed sequence of events [23]. The benefit is understanding that particular blackout, and no probabilistic conclusions are or can be sought. Indeed, statistics cannot be derived from only one sample [24].

\subsection{Mitigation of cascading}

The structure of cascading affects the mitigation strategies for triggers and for propagation. The initiating events can be associated with the cause of the trigger events and the immediately following actions of the protection system. For mitigating the initiating events, the different trigger causes need to be analyzed separately, and there is considerable risk analysis and experience that supports this analysis [17].

Establishing chains of causation in an instance of cascading is useful, but, 
beyond observing that there are multiple dependencies contributing to cascade propagation, there is currently no clear way to attribute causes for complicated cascades. Cascading events are often classified by their root cause, which is the cause of the triggering event. This is useful in mitigating the triggers associated with cascading but root cause analysis does not address the causes or mitigation of propagation. However, it is becoming feasible to relate candidate mitigations such as line upgrades to reductions in propagation or large blackout risk $[25,26$, $27]$.

The emerging capability to quantify the average amount of propagation of cascades [16] opens up the possibility of directly monitoring and mitigating the propagation. The average propagation is independent of the initiating events and is a measure of overall system resilience in the sense that initial outages will on average produce smaller cascades in power systems with lower propagation. There are a variety of mechanisms that contribute to cascade propagation, often entirely different from the mechanisms and causes for the initiating events. Therefore the mitigation of the initiating events and the propagation differ. For example, clusters of lines that outage together more often during propagation can be identified, but these lines can differ from the lines that more often trigger large blackouts $[25,10,26]$.

Since larger blackouts result from both initiating events and the subsequent propagation of events, it is important to monitor and jointly mitigate both the initiating events and the propagation. Decreasing the risk of initiating events while increasing the risk of propagation may not minimize the overall cascading risk. Limiting the triggers and initiating events reduces the frequency of all blackouts, whereas limiting the propagation tends to reduce large blackouts, but may have little effect on the frequency of short cascades.

Over a long time scale, as the power system slowly evolves and upgrades in response to the changing patterns of load and generation, the power system will also respond to any mitigations, and the eventual impact of the mitigation will generally be different than its short term impact. For example, a mitigation initially made to benefit reliability may eventually enable increased transfers that 
bring economic benefits but eliminate the initial reliability benefit [28]. Mitigating small blackouts in the short term can increase the risk of large blackouts in the long term. That is, we can consider the complex system view in which the power system balances both economic pressure to limit upgrade and operational costs and pressure to maintain reliability by investing in upgrades and maximizing transmission. Then a reduction in small blackouts allows economics to drive the system closer to its operational limits and eventually increase the frequency of large blackouts [13]. Thus it is necessary to consider the joint mitigation of small and large blackouts in both the short term and the long term. For example, one can either look for line upgrades that reduce both small and large blackouts in the current power system, or choose sets of line upgrades that have that combined effect. Then one can consider how the benefits of the upgrades evolve as the power system and its operation adapt to the upgrades.

\section{Conclusion}

Analyzing, simulating, and mitigating cascading blackouts in electric power systems poses substantial challenges due to the substantial complexities, dependencies and uncertainties of cascading failure. The current state of the art is to study parts or aspects of the cascading phenomena. In advocating for a more comprehensive approach, it seems timely to state the basic structure of cascading and briefly outline some implications for simulating and mitigating cascading risk. Risk assessment must sample from uncertainties in each of the initial power system state, the initiating events, and the progress of the cascade. The initiating events and the subsequently propagating cascading events that combine to produce large blackouts have different mechanisms, and hence different analyses, and different mitigations. Conventional risk analysis addresses the initiating events well, and there are emerging possibilities to monitor and mitigate the subsequent cascading propagation. These considerations can contribute towards more comprehensive approaches for assessing and mitigating the risk of cascading blackouts. 


\section{Acknowledgements}

We gratefully acknowledge funding in part by the California Energy Commission, Public Interest Energy Research Program and USA National Science Foundation grant CPS-1135825. This paper does not necessarily represent the views of the Energy Commission, its employees or the State of California. It has not been approved or disapproved by the Energy Commission nor has the Energy Commission passed upon the accuracy or adequacy of the information.

\section{References}

[1] IEEE PES CAMS Task Force on Cascading Failure, Initial review of methods for cascading failure analysis in electric power transmission systems, IEEE PES General Meeting, Pittsburgh PA USA, July 2008.

[2] U.S.-Canada Power System Outage Task Force, Final Report on the August 14th blackout in the United States and Canada, United States Department of Energy and National Resources Canada, April 2004.

[3] NERC (North American Electric Reliability Council), 1996 system disturbances, 2002.

[4] Union for the Co-ordination of Transmission of Electricity (UCTE). Final report, system disturbance on 4 November 2006. www.ucte.org.

[5] IEEE PES PSDP Task Force on Blackout experience, mitigation, and role of new technologies, blackout experiences and lessons, Best practices for system dynamic performance, and the role of new technologies, IEEE Special Publication 07TP190, July 2007.

[6] Arizona-Southern California Outages on September 8, 2011: Causes and Recommendations, Federal Energy Regulatory Commission and the North American Electric Reliability Corporation, April 2012. 
[7] I. Dobson, B.A. Carreras, D.E. Newman, A loading-dependent model of probabilistic cascading failure, Probability in the Engineering and Informational Sciences, vol. 19, no. 1, 2005, pp. 15-32.

[8] B.A. Carreras, D.E. Newman, I. Dobson, A.B. Poole, Evidence for self organized criticality in a time series of electric power system blackouts, IEEE Trans. Circuits \& Systems Part I, vol. 51, no. 9, Sept. 2004, pp. 1733-1740.

[9] Q. Chen, C. Jiang, W. Qiu, J.D. McCalley, Probability models for estimating the probabilities of cascading outages in high-voltage transmission network, IEEE Trans. Power Systems, vol. 21, no. 3, Aug. 2006, pp. 14231431.

[10] I. Dobson, B.A. Carreras, D.E. Newman, J.M. Reynolds-Barredo, Obtaining statistics of cascading line outages spreading in an electric transmission network from standard utility data, to appear in IEEE Trans. Power Systems in 2016, available in IEEE Xplore early access.

[11] I. Dobson, B.A. Carreras, V.E. Lynch, D.E. Newman, Complex systems analysis of series of blackouts: cascading failure, critical points, and selforganization, Chaos, vol. 17, no. 2, 026103 June 2007.

[12] P. Hines, J. Apt, S. Talukdar, Large blackouts in North America: historical trends and policy implications, Energy Policy, vol. 37, no. 12, December 2009, pp. 5249-5259.

[13] D.E. Newman, B.A. Carreras, V.E. Lynch, I. Dobson, Exploring complex systems aspects of blackout risk and mitigation, IEEE Trans. Reliability, vol. 60, no. 1, March 2011, pp. 134-143.

[14] B.A. Carreras, D.E. Newman, I. Dobson, North American blackout time series statistics and implications for blackout risk, to appear in IEEE Trans. Power Systems in 2016, available in IEEE Xplore early access. 
[15] R.C. Hardiman, M.T. Kumbale, Y.V. Makarov, An advanced tool for analyzing multiple cascading failures, Eighth International Conference on Probability Methods Applied to Power Systems, Ames Iowa, September 2004.

[16] I. Dobson, Estimating the propagation and extent of cascading line outages from utility data with a branching process, IEEE Trans. Power Systems, vol. 27, no. 4, Nov 2012, pp. 2146-2155.

[17] R. Billinton, R. N. Allan, Reliability Evaluation of Power Systems, second ed. New York: Plenum Press, 1996.

[18] PACME working group of the IEEE PES Reliability, risk and probability applications subcommittee, Effects of dependent and common mode outages on the reliability of bulk electric system - Part I: Basic concepts, IEEE Power and Energy Society General Meeting, July 2014, National Harbor MD USA.

[19] I. Dobson, J. Kim, K. R. Wierzbicki, Testing branching process estimators of cascading failure with data from a simulation of transmission line outages, Risk Analysis, vol. 30, no. 4, 2010, pp. 650-662.

[20] I. Dobson, B.A. Carreras, D.E. Newman, Branching process models for the exponentially increasing portions of cascading failure blackouts, Thirtyeighth Hawaii Intl. Conf. System Sciences, Hawaii, January 2005.

[21] P. Henneaux, P.-E. Labeau, J.-C. Maun, A level-1 probabilistic risk assessment to blackout hazard in transmission power systems, Reliability Engineering \& System Safety, vol. 102, pp. 41-52, 2012.

[22] B.A. Carreras, D.E. Newman, I. Dobson, N.S. Degala, Validating OPA with WECC data, Forty-sixth Hawaii International Conference on System Sciences, Maui, Hawaii, January 2013. 
[23] D.N. Kosterev, C.W. Taylor, W.A. Mittelstadt, Model validation for the August 10, 1996 WSCC system outage, IEEE Trans. Power Systems, vol. 13, no. 3, pp. 967-979, 1999.

[24] I. Dobson, B.A. Carreras, D.E. Newman, How many occurrences of rare blackout events are needed to estimate event probability?, IEEE Trans. Power Systems, vol. 28, no. 3, August 2013, pp. 3509-3510.

[25] B.A. Carreras, D.E. Newman, I. Dobson, Determining the vulnerabilities of the power transmission system, Forty-fifth Hawaii International Conference on System Sciences, Maui, Hawaii, January 2012.

[26] M. Papic, I. Dobson, Comparing a transmission planning study of cascading with historical line outage data, to appear at International Conference on Probability Methods Applied to Power Systems (PMAPS), Beijing China, October 2016.

[27] P.D.H. Hines, I. Dobson, P. Rezaei, Cascading power outages propagate locally in an influence graph that is not the actual grid topology, to appear in IEEE Transactions on Power Systems.

[28] D. Kirschen, G. Strbac, Why investments do not prevent blackouts, Electricity Journal, March 2004, pp. 29-34. 